\title{
Language after dominant hemispherectomy
}

\author{
PEGGY S. GOTT \\ From the Division of Biology, California Institute of Technology, \\ Pasadena, California 91109, U.S.A.
}

SUMMARY Linguistic and related cognitive abilities were investigated two years after dominant left hemispherectomy for cerebral malignancy in a 12 year old female. Auditory comprehension of $\underset{\mathbb{D}}{\mathbb{D}}$ speech was superior to other modes of language abilities with expressive speech being the least $\frac{\Omega}{\omega}$ developed. Findings suggested an isolation or non-communication between the systems for speaking and for writing and visual perception. It was concluded that language mechanisms in the right hemisphere were not just at a low level of development of the functions found in the dominant hemisphere but were modified as a result of interference by preexistent spatioperceptual systems.

Though it is well established that in most righthanded adults the major capacity for language is located in the left hemisphere (Branch et al., 1964; Sperry and Gazzaniga, 1966; Luria, 1970), it is also true that surgical removal of the left cerebral cortex for treatment of infantile hemiplegia results in little if any language deficit (Gardner et al., 1955; Basser, 1962). If brain injury occurs sufficiently early in life, functional plasticity permits the right hemisphere to take over language functions (Krynauw, 1950). The critical age beyond which speech functions cannot be satisfactorily acquired by the opposite hemisphere is said to be about 10 years (Perlstein and Sugar, 1954), but seems variable and has not been sharply determined (Lenneberg, 1967). The exact reason why language fails to develop in the mature brain after hemispherectomy remains unknown.

In a total of 50 or so cases of hemispherectomy for tumour in the mature brain cited in the literature, four of these involved removal of the left hemisphere in right-handed individuals (Zollinger, 1935; Crockett and Estridge, 1951; French et al., 1955; Smith, 1966). In these four adult cases dominant hemispherectomy eliminated expressive language abilities except for expletives, a few stock words, and words of songs.

One case of dominant hemispherectomy for tumour in a 14 year old boy was reported by Hillier (1954), but very few details of the post- operative language abilities of this young boy were reported. It was stated, however, that whereas comprehension of the spoken word was quite accurate, reading ability during the $2 \mathrm{~d}$ month survival period remained limited to the recognition of individual letters; he was unable to read words. Although the upper level of speech that survived in this 14 year old was nat precisely indicated, it would not appear to have been significantly above that found after adu hemispherectomy.

The present report describes some effects of dominant left hemispherectomy in a 12 year old girl in whom earlier removal of a tumour in the left lateral ventricle had been performed at the age of 8 years. The tests described here were mainly designed to determine the level of linguistic and related cognitive abilities in the remaining right hemisphere.

\section{CASE HISTORY}

The subject was a 12 year old girl, R.S., the patient of Dr. P. J. Vogel introduced to the writer through 3 . the courtesy of Dr. J. E. Bogen. At the age of 8 years $\delta$ a tumour was discovered and removed from the left $\xi$ lateral ventricle. Language was not noticeably affected after tumour removal. Reappearance of the malignancy in the parietal area at the age of 10 years necessitated removal of the left hemisphere, which was performed by Professor P. J. Vogel, Chief of Neuro- o surgery at the White Memorial Medical Center in Los N Angeles. Her parents reported that when she awoke N 
from surgery she was conscious and could sing and speak single words, for instance her sister's name.

Before the hemispherectomy R.S. had been righthanded, but the operation left a severe right hemiplegia. Subsequent neurological examination revealed also a severe left side motor deficit of the leg and a mild deficit in the arm (Bogen, 1972). During the present study, two years after surgery, a right homonymous hemianopia with sparing of $1^{\circ}$ or so of the macular region was found.

R.S. had been a slow learner even before the initial brain surgery at the age of 8 years. She had been required to repeat the first grade at the age of 7 years. After removal of the first tumour she returned to school and completed the third grade. Formal

\section{The cat has fur.}

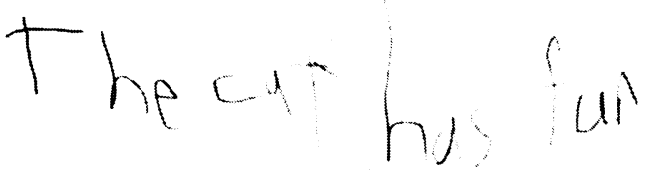

\section{Although it is difficult,}

\section{I can do it.}

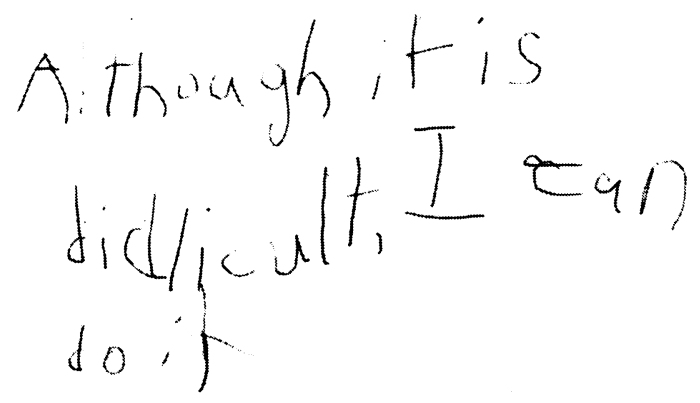

FIG. 1. Examples of R.S.'s handwriting when copying sentences. education was not resumed until a year and a half after hemispherectomy. School records indicate that the Wide Range Achievement Test was given to R.S. in 1968, after removal of the first tumour but before the hemispherectomy. This test indicated that at this time her reading level was first grade, third month, spelling second grade, zero months, and arithmetic second grade, sixth month. Her IQ in 1968 was found to be 86 measured by the StanfordBinet scale. However, before removal of the first tumour her IQ was found to be 100 on the KuhlmannAnderson Intelligence Test administered at school in 1967 and her IQ was 110 on the Lorge-Thorndike Intelligence Test given in 1965 at the beginning of the first grade. Her score on the Wechsler Intelligence Scale for children, administered by the writer at the age of 12 years, was found to be 55 .

Additional observations from the present study indicate that personality characteristics such as humour, boredom, love, and frustration are readily exhibited by the right hemisphere in a pattern reported by the parents to be substantially the same as before surgery. Also, two years after hemispherectomy, it was found that she had learned to draw and to some extent write with her left hand. Figure 1 shows an example of R.S.'s handwriting when she copied sentences presented to her in free vision. A problem with motor control is evident. She was unsuccessful in attempts to write to dictation.

\section{RESULTS}

AUDITORY COMPREHENSION OF SPEECH COMprehension of verbal speech was one of the least impaired of the language functions. Her verbal intelligence measured by the Peabody Picture Vocabulary Test was found to be 70 . The test measures the subject's hearing vocabulary and does not require a verbal response. Smith (1966) reported results which indicate a score of 72 on the same test by E.C., a male with left hemispherectomy for tumour at the age of 47 years. E.C. had a higher ceiling score of 108 , compared with 79 for R.S., which may be correlated with his acquisition of language competence before surgery, while R.S. being much younger had not advanced to the same level preoperatively. This does not necessarily indicate that R.S. was inferior to E.C. in receptive language. R.S. was able to follow most of the directions as tested by the Specific Skills Series modified to auditory instead of visual linguistic input. The requirements of the test included such things as, 'Put an $\mathrm{X}$ on the picture which shows what we sleep in', 
'Draw a cat under the table', or 'Put a circle around the picture of something good to eat'. Superlatives, such as the biggest fish or the smallest dog, were easily identified, and the concept of prepositions, such as without, under, in, and above, were readily understood. Performance became impaired, however, when two or more instructions were included in the same sentence-for example 'Find the boy with the cup and the girl with the bell and draw a line under each'. She would do one part of the task when the directions were first given. However, if they were repeated two or three times she would follow through and perform the entire task.

In addition to the ability to follow verbal directions, R.S. could understand propositional speech and give the proper 'yes' or 'no' answer to statements such as, 'A house can walk', 'A chicken has two feet', 'Baby elephants can read', or 'Rabbits can hop and jump'. She could also choose from a choice of three statements: 'What do firemen do? Bring the milk; Put out fires; Make the bread', or 'What can baby do? Read the book; Play baseball; Sleep and cry'. If, however, the number of such statements is increased to a sequence of four or five to form a short story she cannot properly answer questions about the content of the paragraph after a single reading. This may be more an indication of a lack of memory and mental grasp than a lack of comprehension per se.

Rhyming is easily comprehended by the right hemisphere. R.S. was $100 \%$ correct in determining whether a list of 20 words presented orally rhymed with 'mat'. The concepts of synonyms or antonym words were not understood. Out of 20 pairs of words, 10 pairs which were antonyms and 10 which were unrelated, she was only $40 \%$ correct in determining if two words of a pair were opposite or not. Similarly, with 20 pairs of words, 10 of which were synonyms, her response to whether they were similar or nonsimilar was only $50 \%$ correct.

VOCABULARY R.S.'s scaled score on the Vocabulary Subtest of the Wechsler Intelligence Scale for Children was 8, which put her in the 'dull normal' classification, since the average score on this subtest is 10 . Spelling level measured by the Wide Range Achievement Test was kinder- garten-sixth month. Some insight into the mechanism of verbal analysis of the right hemisphere may be evident from R.S.'s response to the word 'spangled'. She immediately placed her hand over her heart, sang 'God Bless America' in its entirety, then stated, 'Now that is what it is'. The right hemisphere seems capable of making associations and is particularly fond of the musical mode of expression. R.S. particularly likes to sing and does so well, usually with proper words. Expression by singing seems much easier and more fluent than ordinary speech.

TABLE 1

WORDS IN FREE VISION

\begin{tabular}{|c|c|c|c|}
\hline Word & Response & Word & Response \\
\hline Cat & Cat & Dog & Dog \\
\hline Egg & Eat & Three & - \\
\hline Car & -* & Hand & \\
\hline Key & - & Baby & Baby \\
\hline Hat & Hat & Comb & \\
\hline Pen & & Door & - \\
\hline Cup & Coffee, Tea & Lamp & \\
\hline Bed & 一 & Book & Poem \\
\hline Box & - & Coat & - \\
\hline Boy & Boy & Cake & Yum Yum \\
\hline \multicolumn{4}{|c|}{ Blank indicates subject said, 'Don't know'. } \\
\hline
\end{tabular}

SPEECH Speaking was limited as a rule to single words or short phrases. Even so, expressive speech in this subject appeared to be muchō better than that reported by E.C. who was\% unable to speak voluntarily most of the time and $\stackrel{\mathbb{2}}{2}$ in whom propositional speech was only occas- $\overrightarrow{\overrightarrow{0}}$ ionally evident. In contrast, R.S. was able to name correctly $70 \%$ of the pictures shown from the Aphasia Rehabilitation Kit. An additional $20 \%$ of the pictures were identified by verbaliz -5 ing a correct association to the picture-for: example, a picture of a cake was said to be a 3 . 'yum-yum', her response to a picture of a cupd was 'coffee, tea'. If she was then given a choice $₹$ of three or four words presented orally, sheo would correctly indicate the word which named the picture. At other times she visibly strained to을 express the name of an object, like a chair or airplane, and would say, 'I can't'. Again, further' inquiry revealed that she actually did know and 0 
understand the proper word but was unable to bring it to expression. Articulation, not recognition, appeared to be the most difficult component. She is able to repeat words and sentences of six or seven words after they are presented orally with no difficulty, but it is the initiation of verbal expression de novo without auditory assistance which is defective.

RECOGNITION OF WRITTEN MATERIAL Identification of written words was considerably inferior to the recognition of pictures. Only $25 \%$ of the three or four letter words presented visually from the Aphasia Rehabilitation Kit were

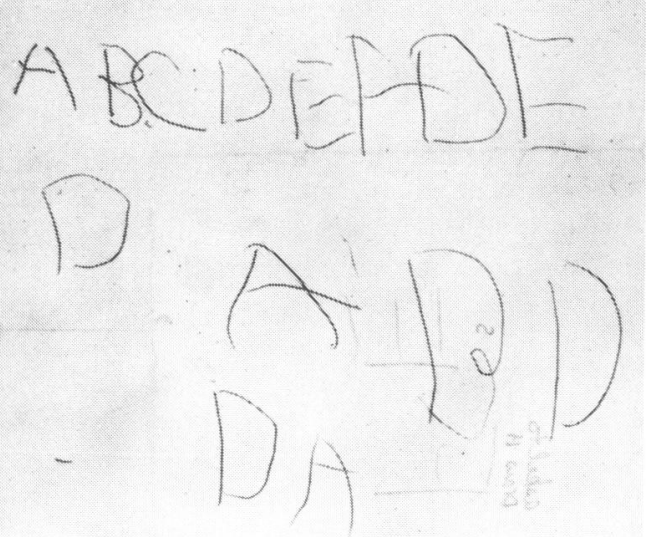

FIG. 2. R.S. attempted to write the alphabet.

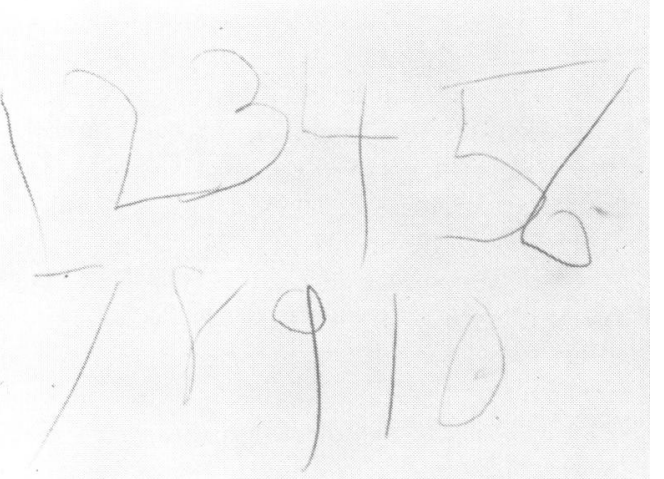

FIG. 3. R.S. wrote numbers from 1 to 10. correctly named (Table 1). However, an additional $20 \%$ were identified by her giving an associative word. For example, she called the word book, 'poem' and she said 'eat' when shown the word 'egg'. On the Wide Range Achievement Test her reading level was only that of kindergarten-seventh month. While the subject could rapidly and accurately repeat the alphabet, she could not correctly name 3-in. plastic letters placed one at a time before her in free vision. Her performance on retrieving an orally designated letter of the alphabet from a choice of four letters was only $31 \%$ correct. Attempts to write the alphabet, even immediately after saying it, were also unsuccessful (Fig. 2). An unexpected result was that she was able to determine if two written words rhymed. Given a list of 20 written words, 10 of which rhymed with 'son' but many of which did not have the same letters, such as sun or bun, and 10 which did not rhyme with 'son' and had some letters in common with son, such as sin and sow, she was $80 \%$ correct in indicating if the word rhymed with son. Yet, the only words she could identify from the list were sun and boy. No comparable tests were reported for E.C.

NUMERICAL ABILITIES R.S. could count correctly to 30 or 40 and could write numbers from 1 to 10 (Fig. 3). If, however, she was then asked to name the number pointed to, the only way she could do so was to begin with one and count until she reached the desired number. She could correctly signal with her fingers the number pointed to, but she had to count her fingers one by one before verbalizing the number she had indicated. The inability to name the number out of sequence was not an indication that she did not know the name of the symbol because using a different sensory route, auditory to visual perception, she was correct $80 \%$ of the time in choosing the called for number from a group of four 3-in. black plastic numbers placed before her.

R.S. could perform simple addition. She selected correct answers to seven out of eight problems (sums of two numbers of one digit) from four possible correct answers. The calculations were performed swiftly and without using aids such as counting her fingers, even though she could not readily name the numbers she was manipulating. However, she could not 
correctly add more than two digits, and her performance on simple subtraction problems involving numbers below 11 was not above chance. Her grade level on the Wide Range Achievement Test for arithmetic was first grade, zero months (1-0). Arithmetical reasoning appeared to be superior in E.C. He was capable of multiplying and making correct calculations with money from 19 cents to $\$ 1 \cdot 17$. Again, the superiority of E.C. may be attributed to his greater knowledge and education before surgery, compared with R.S. R.S.'s mathematical abilities seemed linked primarily to one system, writing. If she was asked verbally or by numeral to count out a certain number of wooden sticks, up to 20 , she was correct on only $30 \%$ of the trials.

TABLE 2

DOTS IN FREE VISION

\begin{tabular}{cccc}
\hline Actual number & Counted & Wrote & Said \\
\hline 6 & 6 & 6 & 1 \\
1 & 1 & 1 & 1 \\
4 & 4 & 4 & 4 \\
10 & 10 & 12 & 4 \\
7 & 7 & 7 & 4 \\
6 & 6 & 7 & 6 \\
1 & 1 & 1 & 1 \\
8 & 8 & 8 & 6 \\
3 & 3 & 3 & 1 \\
8 & 8 & 7 & 8 \\
\hline
\end{tabular}

Additional tests of numerical processes involved the enumeration of dots. R.S. was asked to indicate the number of dots, white dots on a black background, either flashed tachistoscopically or with prolonged exposure on a screen. With tachistoscopic presentation she was asked to signal with her fingers the number of dots and then say the number. Her responses with manual signalling were only $30 \%$ correct, but significantly only five times, $25 \%$ did she verbalize the same number that she had just signalled manually. There was a great deal of perseveration in the verbal report. Likewise, when dots were presented in free vision and she was asked to write and then say the number presented, the verbal report deviated from the written number in $70 \%$ of the responses (Table 2). In this task she counted the dots out loud, always correctly, wrote a number $70 \%$ correct, and then said a number
$40 \%$ correct. When the written and verbal reports did agree, the quantity of the number wase less than five.

COLOUR IDENTIFICATION The proper names could not be determined either verbally or by⿳亠े matching a written word to the colour discs. from the Nomenclature Test of the Dvorine Pseudo-Isochromatic Plates. Yet she could $\stackrel{\text { P }}{+}$ correctly indicate the colour for 14 out of $16 \frac{}{2}$ discs if a choice of several names were given $\frac{\bar{D}}{\bar{\alpha}}$ verbally. She chose the requested colour from a choice of five colours $80 \%$ of the time if the name were spoken orally. E.C., however, picked ${ }^{\text {s }}$ the proper colour if the name were either. written or spoken. There is no indication $\overrightarrow{\vec{\omega}}$ though, that E.C. was able to express the nameo of a given colour. The difficulty in R.S.'s case does not appear to be perceptual, since she was able to match one coloured card to the sameos coloured card in a group of five colours. Her' performance was normal for colour discriming tion on the Dvorine Test. The difference between ${ }_{0}^{N}$ the two cases is probably attributable again $\mathrm{NO}^{\circ}$ the fact that E.C. had the knowledge, in this case colour names, well ingrained before surgery, while R.S. did not. She would usually nates some colour but not the correct name whent presented a colour. Even though visual perce tion of colour was correct, lack of adequate language prevented proper expressing of the colour name.

\section{DISCUSSION}

Two years after the left hemispherectomy at the age of 10 years, tests for auditory compre윽 hension, speech, writing, and other linguistic functions revealed a level of language surpassing that indicated for the four adult left hemi spherectomies described earlier (Zollinger, 1935: Crockett and Estridge, 1951 ; French et al., 1955 ; Smith, 1966). The linguistic capabilities found if. R.S. also surpassed those of the only reallyo comparable case, that of a 14 year old boy (Hillier, 1954) for whom the postoperative period? at the time of testing was also about the same. $\frac{>}{2}$

On the basis of results from dichotic listening tests, Krashen and Harshman (1972) hypothesizes that lateralization of language is completed by the age of 5 years. Little or no language disturb సิ 
ance was reported after left hemisphere lesions which occurred before the age of 10 years (Basser, 1962). Alajouanine and Lhermitte (1965) found recovery from aphasia after left-sided lesions acquired from ages 6 to 15 years; no difference in speed of recovery was found when the group under 10 years old was compared with the group over the age of 10 years. Lenneberg (1967) states that language learning can take place in either hemisphere up to the age of about 13 years. In the foregoing, however, it is uncertain to what extent language may have been dependent on partial preservation of function in the damaged left hemisphere. From the present case, it would appear that the right hemisphere totally unassisted can direct some functions of receptive and expressive language as described above. The exact time at which the right hemisphere in R.S. began to acquire control of linguistic functions cannot be precisely determined but was possibly as early as age 7 or 8 years. The upper level of language achieved after hemispherectomy might have been held down by the low IQ and possible damage to the right hemisphere.

TABLE 3

FUNCTIONAL LANGUAGE CONNECTIONS

\begin{tabular}{lcccc}
\hline & Numbers & Letters & Words & Colours \\
\hline Receptive mode & & & & \\
$\quad$ Auditory & + & + & + & 0 \\
$\quad$ Visual & + & 0 & 0 & + \\
Expressive mode & & & & \\
$\quad \begin{array}{l}\text { Automatic speech } \\
\text { Conscious speech }\end{array}$ & + & + & + & + \\
$\quad$ Writing & 0 & 0 & + & 0 \\
$\quad$ Signalling & + & 0 & 0 & 0 \\
& + & 0 & 0 & + \\
\hline
\end{tabular}

+ Functional. 0 Non-functional. † Partially functional.

The superiority for auditory comprehension as compared with expressive speech, evident in R.S., was also described in the other four reported cases of hemispherectomy for tumour. Difficulty in expressive language in this case cannot be attributed solely to low mentality, since she was able to perform certain tests, Porteus Maze and Block Design, better than two right hemispherectomy cases both with higher IQs (Gott, 1973). Results show that her proficiency in spelling, arithmetic, and reading even two years after hemispherectomy was below the preoperative level. Findings indicated an increasing level of difficulty for visual comprehension beginning with pictures and ascending through numbers and letters to words.

The language systems functioning in R.S.'s minor hemisphere do not appear to represent just a low level of language development as seen in normal acquisition but contain certain abnormalities. The results suggest that her present language system is essentially dissociated from or non-communicating with certain other functions developed in the same hemisphere. The functional relation of the various systems associated with receptive and expressive language found in this case is shown in Table 3. Auditory comprehension was evident for numbers, letters, and the syntax and semantic use of words. On the other hand, visual perception other than spatial perception such as pictures is limited primarily to the recognition of numbers; few letters or words can be identified. The dissociation of automatic verbal habits and consciously directed speech is so great that R.S. is unable to name or write a given letter or number except by reciting the entire sequence. Likewise, what was written was not known by the speech centre, therefore what was said was not what was written.

This functional disconnection between language and other systems within the same hemisphere has not been reported after left hemispherectomy for infantile hemiplegia. In the case of a more mature brain, however, it is necessary for the language system to attempt to take over parts of the brain where differentiation and specialization have already occurred to some extent. The presence of existing organization may prevent the functional connections between language and other systems from becoming properly organized. Levy (1969) finds evidence for an antagonism between linguistic and perceptuospatial functions, the latter being elaborated in the right hemisphere. Whether the mechanism of prevention is competition for existing neural tissue or whether there is actual inhibition to the formation of anatomical connections or to their functioning after they are formed can only be speculated. However, results 
from the present case and other related studies indicate that the effectiveness in preventing the right hemisphere from sustaining language functions increases with age.

I would like to thank Dr. Roger Sperry for helpful suggestions and assistance in preparation of the manuscript and Mary Beth Keppel for help in preparation and administration of the tests. This work was supported by United States Public Health Grant MH-03372 and United States Public Health Fellowship 1 F02 NS 51207.

\section{REFERENCES}

Alajouanine, T., and Lhermitte, F. (1965). Acquired aphasia in childhood. Brain, 88, 653-662.

Basser, L. S. (1962). Hemiplegia of early onset and the faculty of speech with special reference to the effects of hemispherectomy. Brain, 85, 427-460.

Bogen, J. E. (1972). Personal communication.

Branch, C., Milner, B., and Rasmussen, T. (1964). Intracarotid sodium amytal for the lateralization of cerebral speech dominance. Journal of Neurosurgery, 21, 399-405.

Crockett, H. G., and Estridge, N. M. (1951). Cerebral hemispherectomy. Bulletin of the Los Angeles Neurological Societies, 16, 71-87.

French, L. A., Johnson, D. R., Brown, I. A., and Van Bergen, F. B. (1955). Cerebral hemispherectomy for control of intractable convulsive seizures. Journal of Neurosurgery, 12, 154-164.

Gardner, W. J., Karnosh, L. J., McClure, C. C., Jr., and Gardner, A. K. (1955). Residual function following hemispherectomy for tumour and for infantile hemiplegia. Brain, 78, 487-502.

Gott, P. S. (1973). Right and left hemispherectomy. Cortex. (In press.)

Hillier, W. F., Jr. (1954). Total left cerebral hemispherectomy for malignant glioma. Neurology (Minneap.), 4, 718-721.

Krashen, S., and Harshman, R. (1972). Lateralization and the critical period. UCLA Working Papers in Phonetics, 22, 6 .

Krynauw, R. A. (1950). Infantile hemiplegia treated by removing one cerebral hemisphere. Journal of Neurology, Neurosurgery, and Psychiatry, 13, 243-267.

Lenneberg, E. H. (1967). Biological Foundations of Language. Wiley: New York.

Levy, J. (1969). Possible basis for the evolution of lateral specialization of the human brain. Nature, 224, 614-615.

Luria, A. R. (1970). Traumatic Aphasia. Mouton: Hague.

Perlstein, M. A., and Sugar, O. (1954). Hemispherectomy in infantile hemiplegia. Archives of Neurology and Psychiatry, 72, 256-257.

Smith, A. (1966). Speech and other functions after left (dominant) hemispherectomy. Journal of Neurology, Neurosurgery, and Psychiatry, 29, 467-471.

Sperry, R. W., and Gazzaniga, M. S. (1967). Language following surgical disconnection of the hemispheres. In Brain Mechanisms Underlying Speech and Language, pp. 108-121. Edited by F. L. Darley. Grune and Stratton: NewP York.

Zollinger, R. (1935). Removal of left cerebral hemisphere $\frac{\rho}{9}$ Archives of Neurology and Psychiatry, 34, 1055-1064. 